Research Article

\title{
Synaptic Injury in the Thalamus Accompanies White Matter Injury in Hypoxia/Ischemia-Mediated Brain Injury in Neonatal Rats
}

\author{
Na Liu $(\mathbb{D}$, Xin Tong, Wanjie Huang $(\mathbb{D}$, Jianhua Fu $\mathbb{D}$, and Xindong Xue $\mathbb{1}$ \\ Department of Pediatrics, Shengjing Hospital of China Medical University, Shenyang 110004, China \\ Correspondence should be addressed to Xindong Xue; shengjingxxd@vip.sina.com
}

Received 27 May 2019; Revised 15 August 2019; Accepted 13 September 2019; Published 9 October 2019

Academic Editor: Erika Gyengesi

Copyright (C) $2019 \mathrm{Na}$ Liu et al. This is an open access article distributed under the Creative Commons Attribution License, which permits unrestricted use, distribution, and reproduction in any medium, provided the original work is properly cited.

\begin{abstract}
The broad spectrum of disabilities caused by white matter injury (WMI) cannot be explained simply by hypomyelination. Synaptic injury in the thalamus may be related to disabilities in WMI survivors. Neuronal injury in the thalamus has been found most commonly in autopsy cases of preterm WMI. We hypothesized that hypoxia/ischemia (HI) in neonatal rats results in synaptic abnormalities in the thalamus that contribute to disabilities in WMI survivors. We examined changes in synapses in a neonatal rat model of HI-induced WMI. Right common carotid artery ligation and hypoxia ( $8 \%$ oxygen for 2.5 hours (h)) were performed in three-day-old Sprague-Dawley rats. We found HI rats performed worse in the Morris water maze test than sham rats, suggesting long-term cognition impairment after HI injury. A loss of synapses in the thalamus accompanied by hypomyelination and oligodendrocytes (OLs) reduction was observed. At the ultrastructural level, reductions in active zone (AZ) length and postsynaptic density (PSD) thickness were detected at 2 weeks after HI exposure. Furthermore, increased expression of synaptophysin and PSD-95 in both groups was observed from 3 days (d) to $21 \mathrm{~d}$ after hypoxic/ischemic (HI) injury. PSD-95 expression was significantly lower in HI rats than in sham rats from $14 \mathrm{~d}$ to $21 \mathrm{~d}$ after $\mathrm{HI}$ injury, and synaptophysin expression was significantly lower in HI rats from $7 \mathrm{~d}$ to $14 \mathrm{~d}$ after HI injury. However, no significant difference in synaptophysin expression was observed between $\mathrm{HI}$ rats and sham rats at $21 \mathrm{~d}$ after $\mathrm{HI}$ injury. The results demonstrated synaptic abnormalities in the thalamus accompanied by hypomyelination in WMI in response to HI exposure, which may contribute to the diverse neurological defects observed in WMI patients. Although synaptic reorganization occurred as a compensatory response to HI injury, the impairments in synaptic transmission were not reversed.
\end{abstract}

\section{Introduction}

Cerebral white matter injury (WMI) is the leading contributor to neurodevelopmental disabilities in premature infants. Because of improvements in birth and survival rates for premature births in recent years, neurodevelopmental disabilities in survivors have become more prominent [1-3]. In the USA, the neurodevelopmental disabilities for premature neonates with a birth weight less than $1500 \mathrm{~g}$ include cerebral palsy in approximately $10 \%-15 \%$ of the survivors and behavioral deficits in approximately $25-50 \%$ of the survivors, including cognitive decline [2, 4]. Because WMI leads to adverse outcomes and a great social burden, efforts have been made to understand the disease and to reverse brain injury.
Hypomyelination resulted from disrupted synthesis of myelin after injury is commonly found in WMI survivors, especially in diffuse WMI survivors [5-8]. Oligodendrocytes (OLs) degeneration and dysmaturation may contribute to the pathogenesis of hypomyelination [1, 5, 6, 8-10]. Premyelinating oligodendrocytes (pre-OLs), which have been reported to be highly susceptible to $\mathrm{HI}$ and inflammation, have been shown to be the main cell target in WMI $[3,5,9,10]$. The broad spectrum of disabilities caused by WMI, including behavioral deficits (e.g., cognitive, attentional, and motor deficits), appears to be related to not simply myelination failure. The neuroanatomic substrate of diverse disabilities of WMI survivors remains unclear. The thalamus is related to cognition via extensive connections 
with the cerebral cortex [11]. Evidence of neuron loss has been found in autopsy cases of periventricular leukomalacia (PVL), a severe form of preterm WMI [12]. Neurons in the thalamus are most commonly affected in WMI infants [12], potentially contributing to the diverse spectrum of neurological impairments $[7,13]$. Neuroimaging studies indicated a diminished volume of the thalamus in long-term survivors with preterm WMI [14]. Thus, we chose the thalamus as a typical area for research.

Synapses are formed between different neurons and act as the basic unit for information transfer in the brain. Synaptic connections are essential for the organization of the complicated network in the human brain $[15,16]$. Due to their important foundation for interneuronal connection, synapse loss and synaptic dysfunction lead to a series of disorders, including cognitive, learning, attentional, and motor deficits $[16,17]$. Synaptogenesis and plasticity are important in immature brain development [16]. Dysfunction and loss of synapses during immature brain development can result in diverse neurological sequelae, including cognitive, learning, attentional, and motor deficits. Synapse degeneration can be found in the animal model of hypoxic/ ischemic (HI) encephalopathy [18], and alterations in the function and expression of glutamate receptors during the neonatal period, which are components of postsynaptic elements, may contribute to future epileptogenesis [19].

Synapses are formed between neurons. Due to evidence pointing towards neuron injuries in the thalamus of WMI patients and the potential relationship between synaptic dysfunction and the neurological sequelae of WMI survivors, we hypothesized that WMI patients may have synaptic injuries in the thalamus. In this study, we used a neonatal rat model of WMI induced by HI injury to investigate synaptic injuries in the thalamus.

\section{Materials and Methods}

2.1. Animals. All animal experiments were approved by the Animal Ethical Committee of China Medical University, Shenyang, China (2017PS140K). The animal model for WMI was induced according to the method provided by Vannucci et al. [20]. The surgery was performed following inhalation anesthesia. The right common carotid artery of 3-day-old (P3) Sprague-Dawley rats was double-ligated after inhalation of anesthesia, and a cut was made between the ligatures as described previously [21]. After the pups were allowed to recover for 1 hour (h), they were exposed to $2.5 \mathrm{~h}$ of hypoxia $\left(8 \% \mathrm{O}_{2} / 92 \% \mathrm{~N}_{2}\right)$ in a container submerged in a water bath $\left(37^{\circ} \mathrm{C}\right)$ to produce HI injury. After hypoxia exposure, all pups were sent back to their dams until they were sacrificed. The rats in the sham group were only exposed to right common carotid artery isolation without ligation or hypoxia exposure.

2.2. Behavioral Study. The Morris water maze was performed as a behavioral test on days P28-P33 ( $n=10$ each group). The facilities consisted of a circular black tank $(160 \mathrm{~cm}$ in diameter and $60 \mathrm{~cm}$ in depth) and a platform
(12 $\mathrm{cm}$ in diameter) in the first quadrant. The tank was filled with water $\left(24 \pm 0.5^{\circ} \mathrm{C}\right)$ to a level of $1.5 \mathrm{~cm}$ above the top surface of the platform. The navigation test was conducted four times daily for five consecutive days. Rats were placed randomly into the water at each of the four quadrants, facing the wall of the pool. A rat that succeeded in finding the platform within $60 \mathrm{~s}$ was allowed to stay on the platform for $20 \mathrm{~s}$. The time taken to find the platform was defined as escape latency. A rat that failed to find the platform within $60 \mathrm{~s}$ was guided to the platform and allowed to stay there for $20 \mathrm{~s}$. On day 6 , the platform was removed and the probe test was conducted. The rat was placed at the opposite quadrant and allowed to swim for $60 \mathrm{~s}$. The data were recorded by a video tracking system (Shanghai Mobile Datum Ltd, Shanghai City, China). All of the tests were performed by researchers who were blinded to the experimental group.

2.3. Immunohistochemistry. Rats were euthanized at 7 or 14 days (d) after HI injury and perfused with $4 \%$ paraformaldehyde in $0.1 \mathrm{M}$ phosphate buffer before brains were harvested ( $n=5$ each group). The brains were then fixed in a $4 \%$ paraformaldehyde solution for $24 \mathrm{~h}$ at $4^{\circ} \mathrm{C}$ and embedded in paraffin. Coronal sections $(3 \mu \mathrm{m})$ were cut (HM340E; Thermo Fisher Microm, Walldorf, Germany). Tissue sections from brains harvested at $14 \mathrm{~d}$ after HI injury were sequentially deparaffinized in xylene and rehydrated in gradient ethanol solutions. Then, the sections were pretreated with heat-mediated antigen retrieval (EDTA-based pH 9.0 solution) for $20 \mathrm{~min}$. Sections were treated with $3 \%$ hydrogen peroxide for $20 \mathrm{~min}$ at $37^{\circ} \mathrm{C}$ and blocked with goat serum for $30 \mathrm{~min}$ at $37^{\circ} \mathrm{C}$. Sections were incubated at $4^{\circ} \mathrm{C}$ overnight with primary antibody (mouse anti-postsynaptic density (PSD)-95 monoclonal antibody, 1:200, Abcam, USA), followed by incubation with secondary antibody and streptavidin-horseradish peroxidase for $20 \mathrm{~min}$ at $37^{\circ} \mathrm{C}$. Finally, the sections were developed with $3,3^{\prime}$-diaminobenzidine and then dehydrated and mounted in neutral balsam. Images were visualized and photographed by a light microscope (Olympus Corporation, Tokyo, Japan).

2.4. Immunofluorescence. After deparaffinization and heatmediated antigen retrieval (EDTA-based $\mathrm{pH} 9.0$ solution), as previously described, the tissue sections were blocked with goat serum for $30 \mathrm{~min}$ at $37^{\circ} \mathrm{C}$ and then incubated at $4{ }^{\circ} \mathrm{C}$ overnight with primary antibody (mouse anti-myelin basic protein (MBP) monoclonal antibody, 1:400, Abcam, USA; rabbit anti-synaptophysin monoclonal antibody, 1:100, Abcam, USA; goat anti-Olig2 polyclonal antibody, 1:50, R\&D, USA). Sections were washed three times with $0.1 \mathrm{M}$ phosphate buffered saline (PBS) for $5 \mathrm{~min}$ before incubation with secondary antibodies (donkey anti-rabbit IgG, $1: 500$, Abcam, USA; donkey anti-mouse IgG, 1 : 500, Abcam, USA; donkey anti-goat IgG, $1: 200$, Abcam, USA) for $4 \mathrm{~h}$ at room temperature and $4^{\prime}, 6$-diamidino-2-phenylindole (DAPI; 1 : 500, Sigma-Aldrich) sequentially for nuclear staining. Immunofluorescent images were visualized and photographed using a confocal laser-scanning microscope (C1; Nikon, Tokyo, Japan). 
2.5. Western Blotting. At $3,7,14$, and $21 \mathrm{~d}$ after $\mathrm{HI}$ injury, rats were euthanized, and brains were harvested ( $n=5$ each group). Thalamic tissues were isolated from the right hemispheres on ice and stored at $-80^{\circ} \mathrm{C}$. Total protein was extracted from frozen thalamic tissue samples and denatured. Protein samples $(50 \mu \mathrm{g})$ were separated by $4 \%-20 \%$ sodium dodecyl sulfate-polyacrylamide precast gel electrophoresis (Genscript, USA; $140 \mathrm{~V}$ for $1 \mathrm{~h}$ ) and then transferred to polyacrylamide difluoride (PVDF) membranes $(100 \mathrm{~V}$ for $60 \mathrm{~min})$. Membranes were blocked in $5 \%$ nonfat milk for $2 \mathrm{~h}$ before overnight incubation at $4^{\circ} \mathrm{C}$ with primary antibody (mouse anti-PSD-95 monoclonal antibody, $1: 1000$, Abcam, USA; rabbit anti-synaptophysin monoclonal antibody, 1:5000, Abcam, USA; mouse anti-tubulin monoclonal antibody, $1: 2000$, Proteintech, USA). Then, the membranes were incubated with the appropriate secondary antibody (horseradish peroxidase-conjugated goat anti-rabbit or anti-mouse antibody, 1:5000; Proteintech, USA) and developed by enhanced chemiluminescence reagents (Thermo Scientific Pierce; Thermo Fisher Scientific, Waltham, USA). The intensities for all the bands were analyzed by ImageJ software and were normalized to the tubulin signal.

2.6. Electron Microscopy. At $14 \mathrm{~d}$ after $\mathrm{HI}$ injury, rats $(n=5$ each group) were euthanized, brains were dissected, and thalamic tissue sections from the right hemispheres were cut to a size of approximately $1 \mathrm{~mm}^{3}$ at 4.0 to $5.0 \mathrm{~mm}$ posterior to bregma. Tissue samples were postfixed with $4 \%$ paraformaldehyde and $2.5 \%$ glutaraldehyde. Then, brain samples were treated in $1 \%$ osmium tetroxide, dehydrated in an acetone series, and embedded with epoxy resin. The tissue blocks were cut into ultrathin sections with an ultramicrotome and stained with uranyl acetate and lead citrate. Images were observed using a transmission electron microscope (JEM-1400; JEOL, Tokyo, Japan). Images were randomly selected and analyzed using ImageJ software.

2.7. Statistics. All the data from each experiment are represented as the mean \pm SEM. GraphPad Prism 5 was used to create graphs and perform statistical analyses. Any significant differences between two groups were assessed by the $t$ test. The escape latency was analyzed using two-way analysis of variance for repeated measurements. The differences were considered statistically significant at $P<0.05$.

\section{Results}

3.1. Impaired Cognition after Exposure to HI. The Morris water maze is widely used for the assessment of spatial learning and memory, which are related to cognitive function [22]. In the navigation test, during the first 5 days, significantly longer escape latency was observed in rats from the HI group than the sham group (Figure 1 (a), $P<0.001$, at $2 \mathrm{nd}, 3 \mathrm{rd}, 4^{\text {th }}$, and 5 th days). In addition, in the probe trial, rats in the HI group passed less frequently through the platform location $(P<0.01)$ and spent less time in the target quadrant $(P<0.05)$ compared to the sham group
(Figures 1(b) and 1(c)), indicating the long-term cognitive deficits of HI rats.

3.2. OL Reduction and Hypomyelination Induced by HI Injury. We performed immunofluorescent staining of subcortical white matter with Olig2 at $7 \mathrm{~d}$ after $\mathrm{HI}$ injury to determine the distribution of Olig2-labeled OLs. As shown in Figures 2(a) and 2(b), the density of Olig2-labeled OLs in the subcortical white matter of right hemispheres was lower in HI rats than in sham rats, which was related to the myelination disturbance in white matter after HI injury.

To determine the effect of $\mathrm{HI}$ injury on myelination, we performed immunofluorescent staining for MBP, a myelin protein. Corpus callosum (CC) is rich in myelin in the periventricular region. It is widely used as a proxy of hypomyelination in perinatal WMI. At $14 \mathrm{~d}$ after $\mathrm{HI}$ injury, $\mathrm{HI}$ rats exhibited less myelination of CC axons in the right hemispheres than did sham rats (Figures 2(a), 2(c), and $2(d))$. The projection fibers oriented to the cortex in the boxed area were hypomyelinated in HI rats, indicating reduced velocity of impulse conduction in this area in HI rats compared to that in sham rats.

3.3. Synaptic Changes Induced by HI Injury. We explored the synaptic changes caused by HI injury at the ultrastructural and biochemical levels.

3.3.1. Changes in the Structure and Numerical Density of Synapses in the Thalamus after HI Injury. Sections of the thalamus at $14 \mathrm{~d}$ after $\mathrm{HI}$ injury were analyzed by electron micrography. Changes in the morphology and density of synapses were investigated at the ultrastructural level.

The active zone (AZ), which is located in the presynaptic membrane, is considered to be involved in transmitter release. The PSD is a series of proteins in the postsynaptic membrane that plays a crucial role in signal exchange between neurons. We analyzed changes in AZ length and PSD thickness of synapses induced by $\mathrm{HI}$ injury. As shown in Figures 3(a)-3(e), compared with the sham group, the HI group exhibited a decrease in both AZ length and PSD thickness.

Compared to the sham group, the HI group exhibited fewer synaptic vesicles at the presynaptic terminal accompanied by a decrease in vesicles located in the $\mathrm{AZ}$ and ready to release (Figures 3(a) and 3(b)).

In the thalamus, the numerical density of synapses in the HI group $(48,343 \pm 7,621)$ was obviously lower than that in the sham group $(80,486 \pm 5,675$, Figure $3(f))$.

3.3.2. Changes in Presynaptic and Postsynaptic Protein Expression. To examine the differential expression of presynaptic and postsynaptic proteins in the thalamus induced by $\mathrm{HI}$ injury, we performed western blotting analysis. After HI exposure, both synaptophysin and PSD-95 showed increasing trends in expression from $3 \mathrm{~d}$ to $21 \mathrm{~d}$ after $\mathrm{HI}$ injury, which indicated continuous synaptogenesis (Figures 4(a)4(d)). The level of synaptophysin was not significantly 


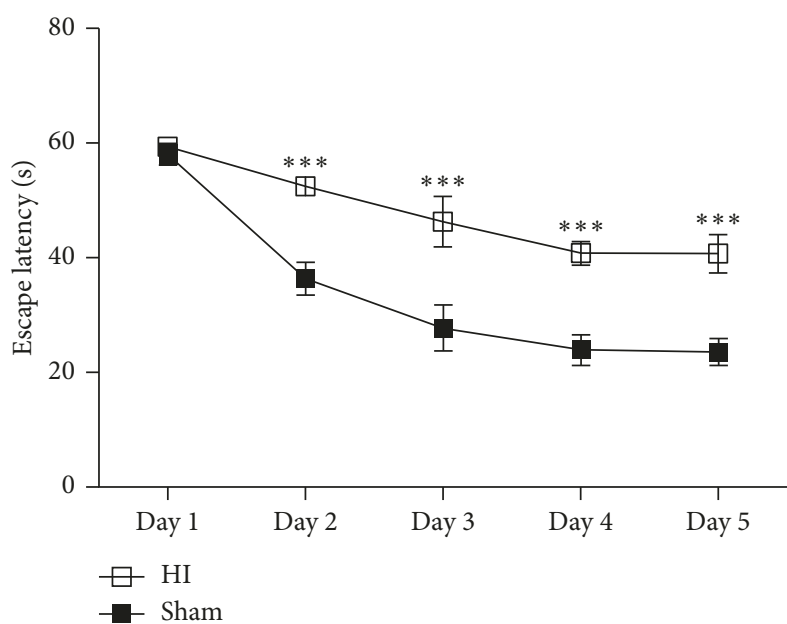

(a)

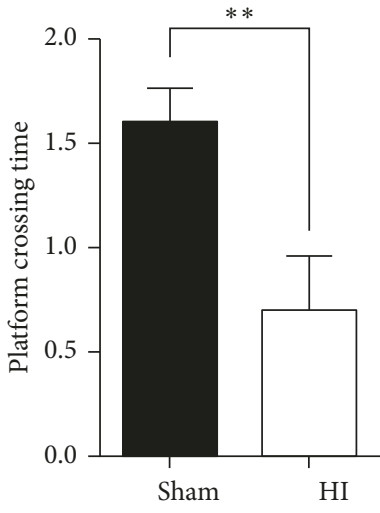

(b)

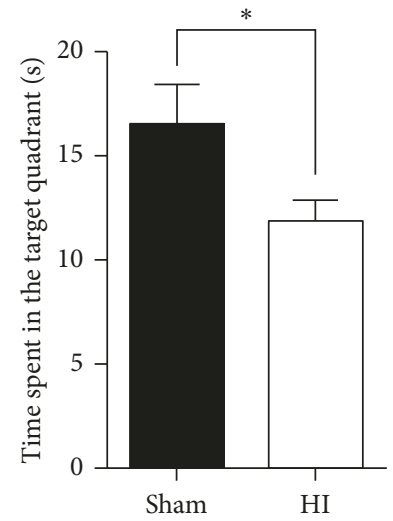

(c)

FIgURE 1: Cognitive function assessment by the Morris water maze test. (a) During the navigation test, HI rats showed a significantly longer escape latency compared to the sham rats from the 2 nd day to the 5 th day. In the probe test, the HI rats showed less platform crossing (b) and spent less time in the target quadrant (c) compared to the sham rats $\left({ }^{*} P<0.05,{ }^{* *} P<0.01\right.$, and $\left.{ }^{* * *} P<0.001\right)$. Two-way analysis of variance (ANOVA) for repeated measurements was used to determine the difference of escape latency. Student's $t$-test was performed to determine the differences in the number of platform crossing and time spent in the target quadrant.

different at $3 \mathrm{~d}$ after $\mathrm{HI}$ injury between the $\mathrm{HI}$ and sham groups. At $7 \mathrm{~d}$ after HI injury, the HI group exhibited significantly lower synaptophysin levels than the sham group $(P<0.05)$, and the difference became more obvious at $14 \mathrm{~d}$ after HI injury $(P<0.01)$. From $14 \mathrm{~d}$ to $21 \mathrm{~d}$ after $\mathrm{HI}$ injury, there was a marked increase in synaptophysin, resulting in no significant difference between the two groups at $21 \mathrm{~d}$ after HI injury. However, the level of PSD-95 significantly decreased at 14 and $21 \mathrm{~d}$ after $\mathrm{HI}$ injury in the HI group, and no difference in PSD-95 expression between the two groups was identified at $3 \mathrm{~d}$ and $7 \mathrm{~d}$ after $\mathrm{HI}$ injury. The difference in time-dependent protein expression indicated that PSD-95 synthesis was later than that of synaptophysin during synaptogenesis.

We also conducted immunofluorescent examination on synaptophysin and immunohistochemical examination on PSD-95 at $14 \mathrm{~d}$ after HI injury. It revealed a significant decrease in fluorescence intensity of synaptophysin in the thalamus of $\mathrm{HI}$ rats compared to sham rats $(P<0.0001$, Figures 5(a) and 5(b)). The analysis of ODs indicated the lower expression level of PSD-95 in the thalamus of HI rats compared to sham rats $(P<0.05$, Figures $5(\mathrm{c})$ and $5(\mathrm{~d}))$.

\section{Discussion}

The degeneration and impaired maturation of OLs in the developing brain have been considered to be the main mechanisms of myelination failure associated with WMI induced by HI injury $[1,6-8,10]$. The present study showed that $\mathrm{HI}$ exposure in rats caused hypomyelination and OLs reduction, in agreement with results from other WMI studies $[23,24]$. The brain region analyzed for the expression of MBP was CC, which was rich in myelin in the periventricular region. In perinatal WMI, decreased expression of MBP was considered to be associated with long-term cognitive dysfunction [25]. The lower MBP expression level was associated with axonal myelination insufficiency determined by electron micrography [26]. Hypomyelination in the CC and cortex will result in insufficient information processing and conduction velocity between cortical regions or between cortex and deep gray nuclei, such as the connections between the thalamus and cortex, which will be discussed in the following part. We further noted loss of synapses in the thalamus. In addition, ultrastructural synaptic features, such as AZ length and PSD thickness, were reduced at 2 weeks after HI injury.

Synapses play a vital role in information transduction between neurons and are essential for complicated signal processing and network organization in the nervous system $[15,16]$. Impaired synapse physiology is involved in the pathogenesis of many neurodevelopmental and neurodegenerative diseases, such as autism [27], Down syndrome [28], epilepsy [29], Alzheimer disease [30, 31], and Parkinson disease [32]. In the present study, we noted a reduction in the numerical density and an increase in ultrastructural defects of synapses in the thalamus of rats with WMI induced by HI injury, probably leading to physiological and behavioral dysfunction [16, 27-33]. In addition, the thalamus has extensive connections with cortical regions and serves as a relay station for sensory information transduction to the cortex, participating in vision formation [11,34]. The network connecting the thalamus and cortex displays regulatory properties in learning, memory, decision-making, and other cognitive behaviors $[11,35,36]$. The importance of connections was highlighted for its association with neurological disabilities [37]. The injury to the thalamus may involve multiple developmental domains, which is consisted with the neurological disabilities including adverse neurocognitive outcomes, visual dysfunction, disturbances in learning, 

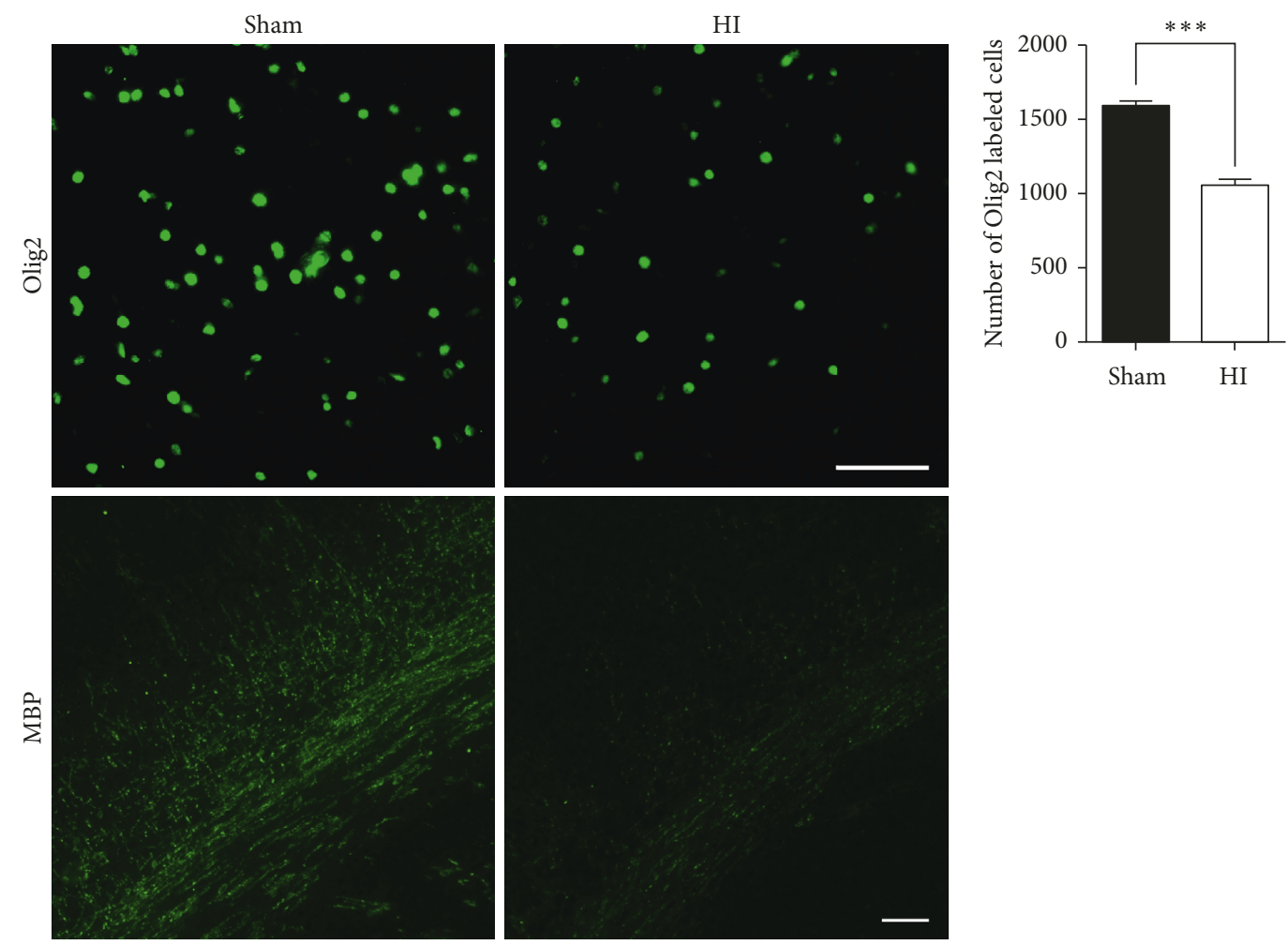

(a)

(b)
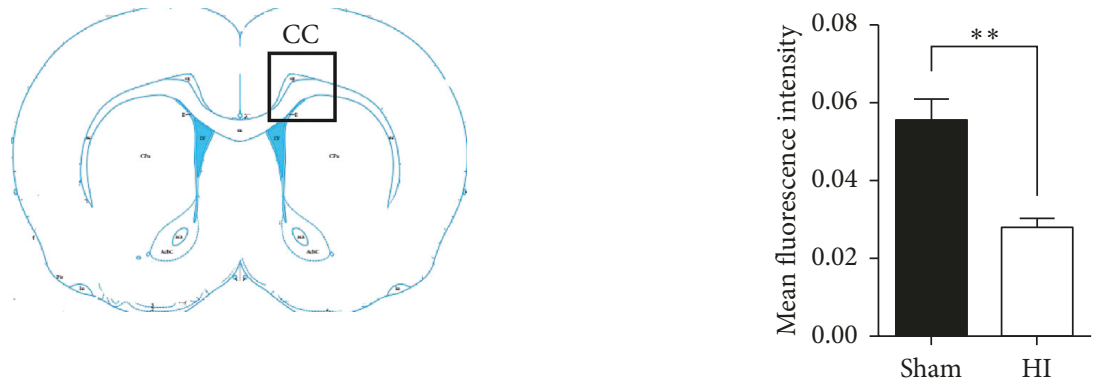

(c)

(d)

FIgURE 2: The changes in number of oligodendrocytes and myelination. (a, b) Olig2+ cells in immunofluorescence staining referred to oligodendrocytes (scale bar $=50 \mu \mathrm{m}$ ). The quantitative analysis showed decreased number of oligodendrocytes $\left(\right.$ per $\left.\mathrm{mm}^{2}\right)$ in subcortical white matter of right hemispheres in HI rats at $7 \mathrm{~d}$ after $\mathrm{HI}$ injury compared with sham rats. (c) Diagram of the brain region analyzed for the expression of MBP. (a, d) Immunofluorescence staining showed MBP in corpus callosum (CC) axons in the right hemispheres (boxed area in (c)) at $14 \mathrm{~d}$ after $\mathrm{HI}$ injury (scale bar $=50 \mu \mathrm{m}$ ). HI rats exhibited less myelination of CC axons in the right hemispheres than sham rats did. The projection fibers oriented to the cortex in the boxed area were hypomyelinated in HI rats $\left({ }^{* *} P<0.01\right.$ and $\left.{ }^{* * *} P<0.001\right)$.

memory, attention, and executive function in WMI survivors that cannot simply be induced by myelination failure. Hypomyelination accompanied by synaptic defects in the thalamus may provide a more comprehensive and reasonable pathological basis for the broad spectrum of disabilities observed in these patients. Thus, we choose the thalamus as a typical area for research, focusing on the synaptic defects in the thalamus. However, neuronal degeneration in the hippocampus and cerebellum was reported in some neuroimaging and neuropathological studies $[12,37]$. The relation of the hippocampus and cerebellum deficits after HI injury with cognitive disability in WMI survivors needs further exploration.
Presynaptic membranes are primarily located on axon terminals, while postsynaptic membranes are located on dendritic spines. McClendon et al. found neuronal dysmaturation in response to $\mathrm{HI}$ injury in the caudate nucleus of fetal sheep, which manifested as a decrease in dendritic spines without neuronal death [38]. By performing quantitative volumetric and DTI measurements, Nagasunder et al. reported a volumetric reduction in the thalamus and thalamic microstructural abnormalities in PVL survivors, suggesting axon degeneration that likely contributed to cognitive visual processing disabilities [14]. The above two findings provide possible anatomic evidence for abnormal synaptic abnormalities in patients with WMI. 
Sham

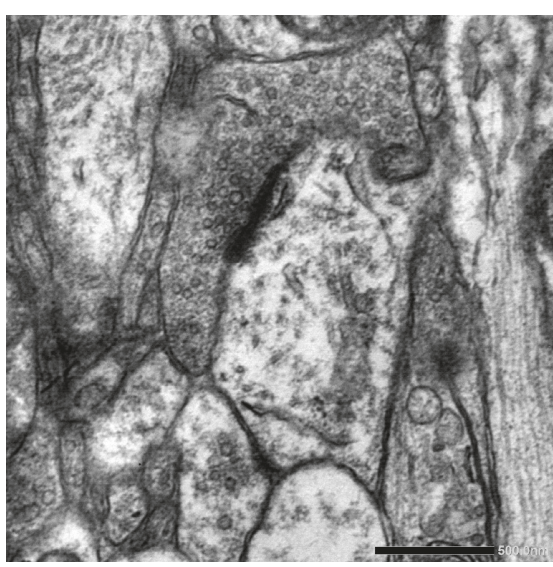

(a)

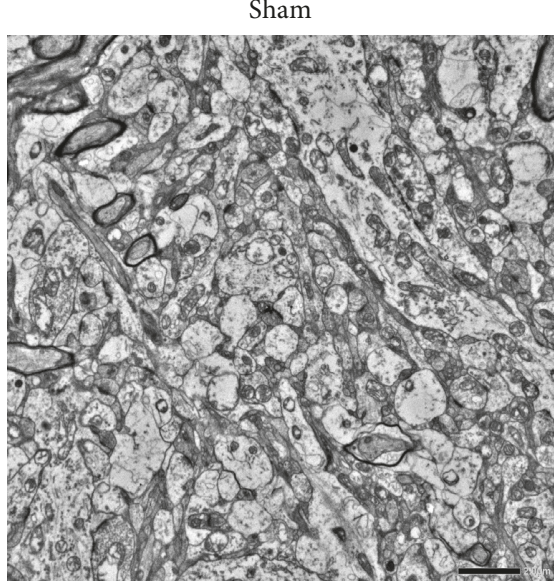

(c)
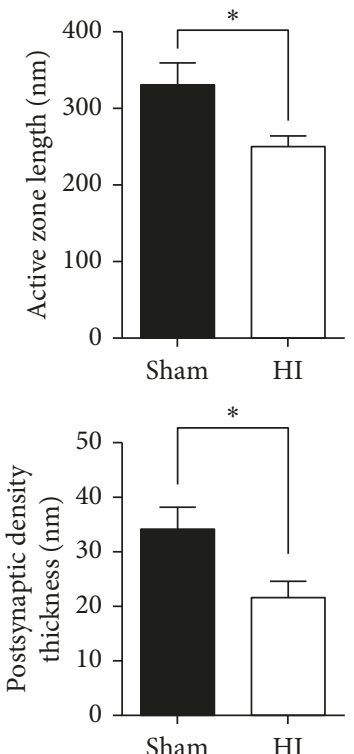

(e)
HI

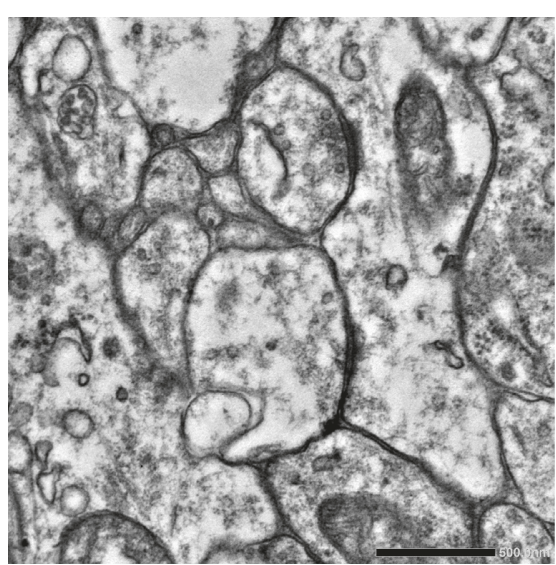

(b)

$\mathrm{HI}$

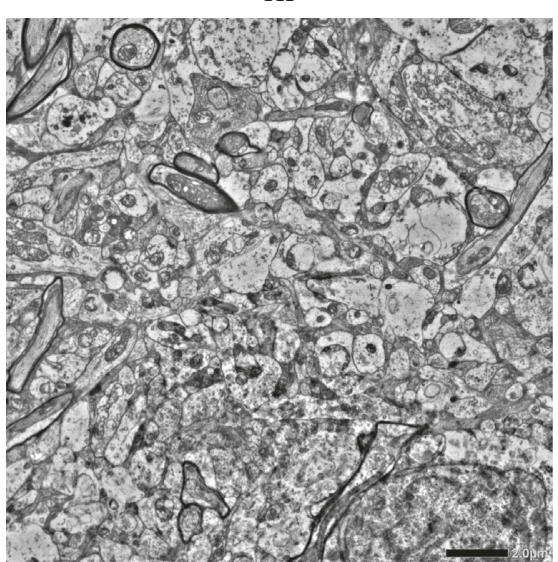

(d)

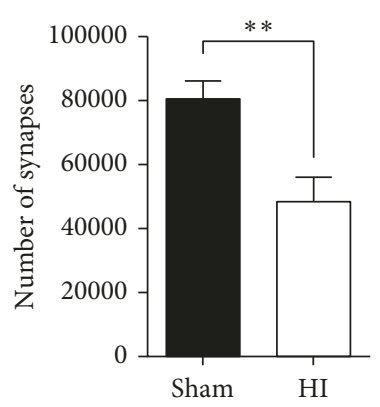

(f)

FIGURE 3: Ultrastructural changes observed in synapses at $14 \mathrm{~d}$ after HI injury in the thalamus. $(\mathrm{a}, \mathrm{b})$ TEM images of the synapses in the thalamus from the right hemispheres exhibited fewer synaptic vesicles at the presynaptic terminal in the HI group, accompanied by a decrease in vesicles located in the $\mathrm{AZ}$ and ready to release (scale bar $=500 \mathrm{~nm}$ ). (c, d) Low magnification of TEM images of the synapses in the thalamus from the right hemispheres recognizable by their postsynaptic density (scale bar $=2 \mu \mathrm{m}$ ). (e) The quantitative analysis showed that the AZ length and the PSD thickness were both decreased in the HI group compared with the sham group at $14 \mathrm{~d}$ after $\mathrm{HI}$ injury in the right thalamus. (f) The quantitative analysis of the numerical synaptic density of synapses per $\mathrm{mm}^{2}$ showed significant decrease of synapses in the right thalamus in the $\mathrm{HI}$ group at $14 \mathrm{~d}$ after $\mathrm{HI}$ injury $\left({ }^{*} P<0.05\right.$ and $\left.{ }^{* *} P<0.01\right)$. 


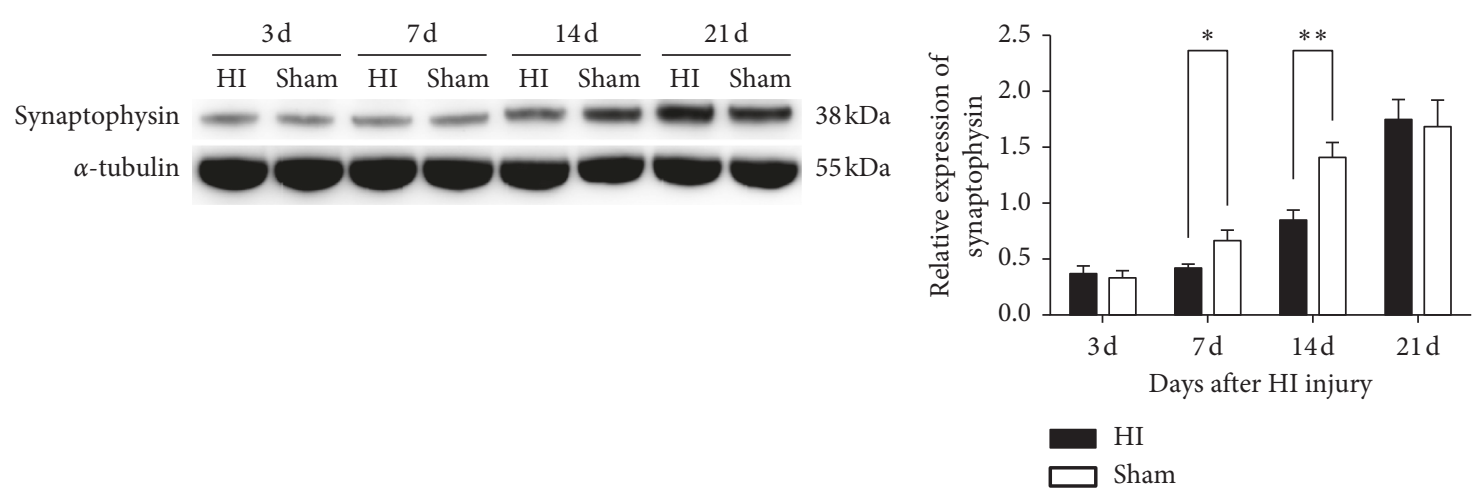

(a)

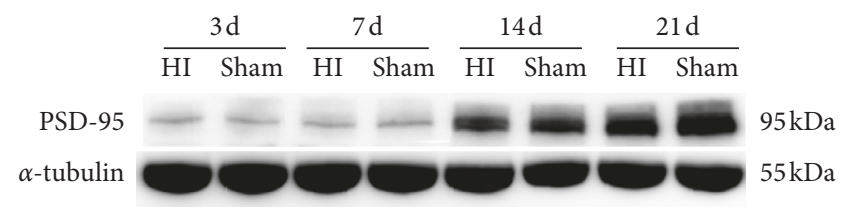

Extracellular matrix (ECM) molecules from reactive astrocytes are viewed as chemical barriers for synaptogenesis and synaptic plasticity $[17,39]$. ECM molecules play vital roles in the early development of the nervous system by guiding neurite outgrowth, synaptogenesis, and synaptic plasticity. In WMI, accumulated reactive astrocytes surrounding the damaged area form glial scars and secrete neurotoxins and ECM molecules, among which hyaluronic acid (HA) and some chondroitin sulfate proteoglycans (CSPGs) have been found to be responsible for limiting neurite growth and axonal regeneration after injury, thereby impairing synaptogenesis and synaptic plasticity [39, 40]. Increased activity of the ECM molecule MMP9 has been associated with structural alterations of spines or altered synaptic plasticity [41]. In addition to ECM molecules, glycogen synthase kinase $3 \beta$ (GSK-3 $\beta$ ) has been shown to be involved in $\mathrm{HI}$ injury-induced synaptic abnormalities. Increased GSK-3 $\beta$ levels after ischemia and reperfusion exposure in rat brains were related to negative regulation of antioxidant ability and neuron death, suggesting possible relationships with synaptic abnormalities [42]. In addition, increased activity of GSK-3 $\beta$ likely promotes tau phosphorylation and neurodegeneration and, subsequently, synapse dysfunction $[43,44]$.
Ultrastructural changes in synapses, manifesting as decreased AZ length and PSD thickness at 2 weeks after HI injury exposure, were shown in the present study. The AZ, which is located on the presynaptic terminal, consists of scaffold proteins ensuring fast but adjustable neurotransmitter release, thus playing a prominent role in regulating the efficacy of neurotransmitter release $[17,45,46]$. In contrast, the PSD is formed by the scaffold of densely organized proteins that anchor neurotransmitter receptors and cell-adhesion molecules responsible for information transduction $[47,48]$. The decreased AZ length and PSD thickness in the thalamus in the present study can be explained by HI injury and suggest impaired interneuronal connectivity and information transduction, which may subsequently result in motor defects or cognitive disability.

We found increased expression of synaptophysin and PSD-95 from $3 \mathrm{~d}$ to $21 \mathrm{~d}$ after HI injury in this study, suggesting continuous synaptogenesis during this period. The two groups demonstrated no significant difference in synaptic proteins expression at $3 \mathrm{~d}$ after injury. One of the possibilities is that damage to axon, which contains the presynaptic protein, may be induced by degeneration secondary to the death of neuronal cell bodies of thalamus [49]. The secondary injury may not be evident during the early 


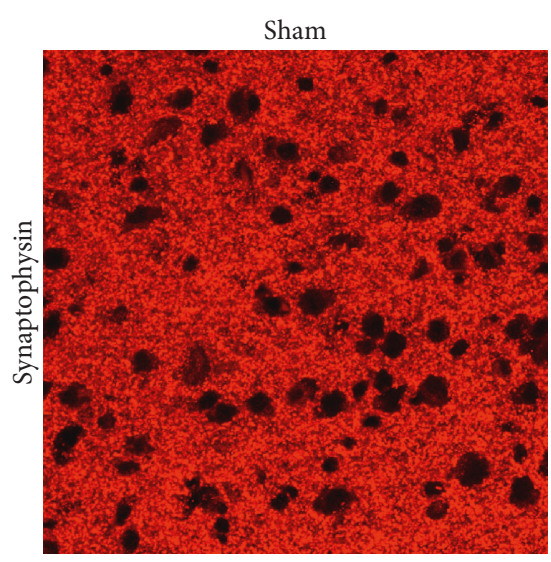

(a)

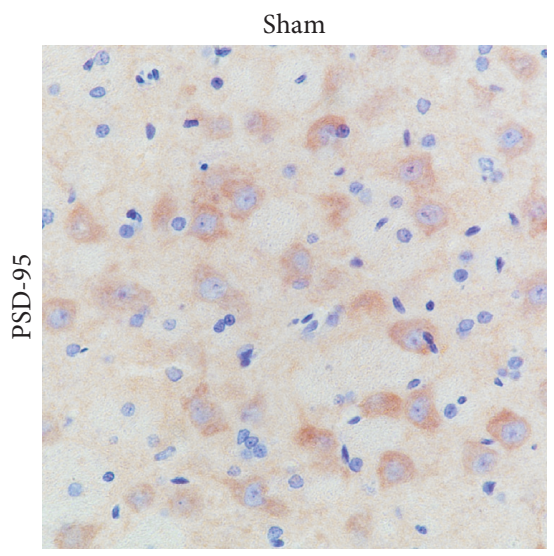

(c)

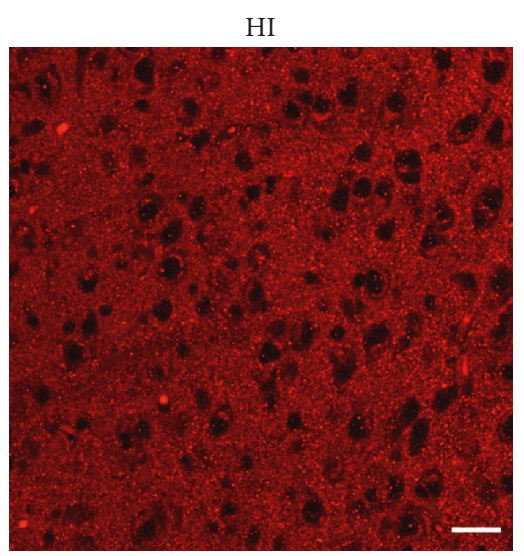

HI

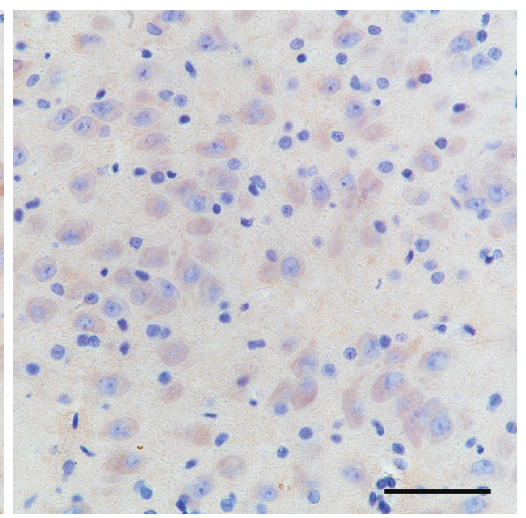

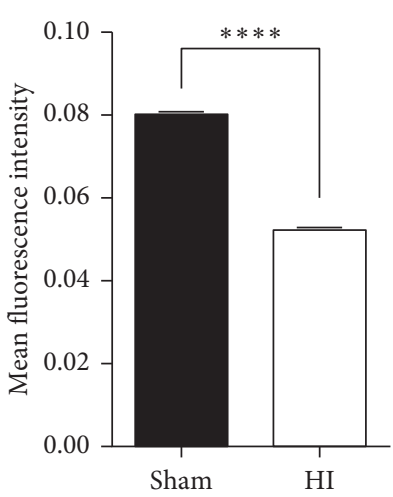

(b)

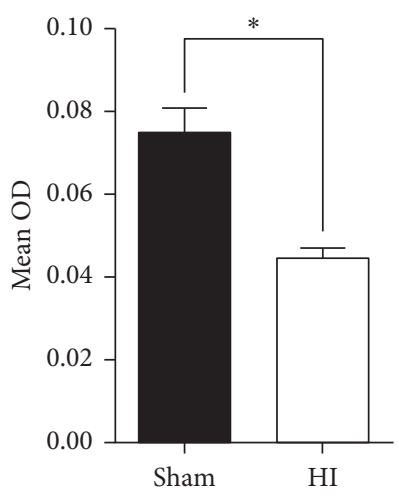

(d)

FIGURE 5: The changes of synaptophysin and PSD-95 expression in the thalamus exhibited by immunofluorescence and immunohistochemistry staining. ( $\mathrm{a}, \mathrm{b})$ Immunofluorescence staining showed synaptophysin in the right thalamus at $14 \mathrm{~d}$ after $\mathrm{HI}$ injury (scale bar $=20 \mu \mathrm{m}$ ). The quantitative analysis showed lower expression of the synaptophysin in the thalamus in HI rats than in that of sham rats. (c, d) Immunohistochemistry staining showed PSD-95 in the right thalamus at $14 \mathrm{~d}$ after HI injury (scale bar $=50 \mu \mathrm{m}$ ). The quantitative analysis showed lower expression of the PSD-95 in the thalamus in $\mathrm{HI}$ rats $\left({ }^{*} P<0.05\right.$ and $\left.{ }^{* * * *} P<0.0001\right)$.

days after HI injury. The delayed injury is important to preterm brain injury, as it results in impairments in the neonate that evolve into complicated disabilities over time [37]. Besides, dendritic spines, which contain the postsynaptic protein, are less in the immature neurons during the early 10 days after birth [50], consisted with the low expression of PSD-95 in sham rats from $3 \mathrm{~d}$ to $7 \mathrm{~d}$ after modelling in the present study. The injury to the spine is probably not significantly manifested at $3 \mathrm{~d}$ after injury. However, the HI group expressed significantly less of these two synaptic proteins at $14 \mathrm{~d}$ after injury than the sham group. The loss of synapses and decrease in AZ length and PSD thickness induced by HI injury provides some possible evidence for the above findings. PSD-95 is a member of the scaffold proteins in the PSD and is involved in the organization of postsynaptic signals and regulation of synaptic transmission [47, 48, 51]. The first identified synaptic vesicle (SV) membrane protein, synaptophysin, is required in the biogenesis of SVs and in the regulation of SV trafficking, which may be related to synaptic plasticity [45]. The decreased levels of synaptophysin and PSD-95 suggest impaired synaptic plasticity and signal transmission. The decreased expression of PSD-95 continued until $21 \mathrm{~d}$ after $\mathrm{HI}$ injury, at which point the levels of synaptophysin were not significantly different between the HI and sham groups. We proposed that the marked increase in synaptophysin from $14 \mathrm{~d}$ to $21 \mathrm{~d}$ after HI injury in HI rats may be a compensatory change that helps to improve the efficiency of transmitter release by promoting the biogenesis of SVs. However, this synaptic reorganization with an imbalance in presynaptic and postsynaptic protein expression, which has also been observed in immature brains with seizure-related injury [52], probably leads to uncoordinated synaptic transmission.

Hypomyelination in the thalamus was reported in some neuroimaging studies $[14,37]$. We have not focused on the change of myelin in the thalamus in the present study. It could be a direction for our future research.

In the present study, we used a rat model of WMI and demonstrated that neonatal $\mathrm{HI}$ injury not only induced $\mathrm{OL}$ reduction and hypomyelination but also led to synaptic loss and ultrastructural and synaptic protein expression abnormalities. Importantly, we found synaptic reorganization induced by HI injury, which may aggravate synaptic 
dysfunction by disturbing signal transmission. Further investigations are needed to explore the mechanism of synaptic reorganization induced by $\mathrm{HI}$ injury and synaptic changes after HI injury over a longer period.

\section{Data Availability}

The data used to support the findings of this study are available from the corresponding author upon request.

\section{Conflicts of Interest}

The authors declare that they have no conflicts of interest.

\section{References}

[1] C. M. Elitt and P. A. Rosenberg, "The challenge of understanding cerebral white matter injury in the premature infant," Neuroscience, vol. 276, pp. 216-238, 2014.

[2] J. J. Volpe, "The encephalopathy of prematurity-brain injury and impaired brain development inextricably intertwined," Seminars in Pediatric Neurology, vol. 16, no. 4, pp. 167-178, 2009.

[3] S. A. Back, "Cerebral white and gray matter injury in newborns: new insights into pathophysiology and management," Clinics in Perinatology, vol. 41, no. 1, pp. 1-24, 2014.

[4] J. J. Volpe, "Encephalopathy of prematurity includes neuronal abnormalities," Pediatrics, vol. 116, no. 1, pp. 221-225, 2005.

[5] A. M. Kaindl, G. Favrais, and P. Gressens, "Molecular mechanisms involved in injury to the preterm brain," Journal of Child Neurology, vol. 24, no. 9, pp. 1112-1118, 2009.

[6] S. A. Back, "Brain injury in the preterm infant: new horizons for pathogenesis and prevention," Pediatric Neurology, vol. 53, no. 3, pp. 185-192, 2015.

[7] J. J. Volpe, "Brain injury in premature infants: a complex amalgam of destructive and developmental disturbances," The Lancet Neurology, vol. 8, no. 1, pp. 110-124, 2009.

[8] J. M. Dean, L. Bennet, S. A. Back, E. McClendon, A. Riddle, and A. J. Gunn, "What brakes the preterm brain? An arresting story," Pediatric Research, vol. 75, no. 1-2, pp. 227-233, 2014.

[9] S. A. Back and S. P. Miller, "Brain injury in premature neonates: a primary cerebral dysmaturation disorder?," Annals of Neurology, vol. 75, no. 4, pp. 469-486, 2014.

[10] O. Khwaja and J. J. Volpe, "Pathogenesis of cerebral white matter injury of prematurity," Archives of Disease in Childhood-Fetal and Neonatal Edition, vol. 93, no. 2, pp. F153F161, 2008.

[11] K. Hwang, M. A. Bertolero, W. B. Liu, and M. D’Esposito, "The human thalamus is an integrative hub for functional brain networks," The Journal of Neuroscience, vol. 37, no. 23, pp. 5594-5607, 2017.

[12] C. R. Pierson, R. D. Folkerth, S. S. Billiards et al., "Gray matter injury associated with periventricular leukomalacia in the premature infant," Acta Neuropathologica, vol. 114, no. 6, pp. 619-631, 2007.

[13] H. C. Kinney, "The encephalopathy of prematurity: one pediatric neuropathologist's perspective," Seminars in Pediatric Neurology, vol. 16, no. 4, pp. 179-190, 2009.

[14] A. C. Nagasunder, H. C. Kinney, S. Blüml et al., "Abnormal microstructure of the atrophic thalamus in preterm survivors with periventricular leukomalacia," American Journal of Neuroradiology, vol. 32, no. 1, pp. 185-191, 2011.
[15] X. Jiang, S. Shen, C. R. Cadwell et al., "Principles of connectivity among morphologically defined cell types in adult neocortex," Science, vol. 350, no. 6264, Article ID aac9462, 2015.

[16] T. J. Ryan and S. G. N. Grant, "The origin and evolution of synapses," Nature Reviews Neuroscience, vol. 10, no. 10, pp. 701-712, 2009.

[17] K. Lepeta, M. V. Lourenco, B. C. Schweitzer et al., "Synaptopathies: synaptic dysfunction in neurological disorders-a review from students to students," Journal of Neurochemistry, vol. 138, no. 6, pp. 785-805, 2016.

[18] T. Xiong, Y. Qu, H. Wang et al., "GSK-3 $\beta / \mathrm{mTORC} 1$ couples synaptogenesis and axonal repair to reduce hypoxia ischemiamediated brain injury in neonatal rats," Journal of Neuropathology \& Experimental Neurology, vol. 77, no. 5, pp. 383-394, 2018.

[19] C. Zhou, H. Sun, P. M. Klein, and F. E. Jensen, "Neonatal seizures alter NMDA glutamate receptor GluN2A and 3A subunit expression and function in hippocampal CA1 neurons," Frontiers in Cellular Neuroscience, vol. 9, 2015.

[20] R. C. Vannucci, J. R. Connor, D. T. Mauger et al., "Rat model of perinatal hypoxic-ischemic brain damage," Journal of Neuroscience Research, vol. 55, no. 2, pp. 158-163, 1999.

[21] T. Cheng, X. Xue, and J. Fu, "Effect of OLIG1 on the development of oligodendrocytes and myelination in a neonatal rat PVL model induced by hypoxia-ischemia," Molecular Medicine Reports, vol. 11, no. 4, pp. 2379-2386, 2015.

[22] C. V. Vorhees and M. T. Williams, "Morris water maze: procedures for assessing spatial and related forms of learning and memory," Nature Protocols, vol. 1, no. 2, pp. 848-858, 2006.

[23] S. A. Back, N. L. Luo, R. A. Mallinson et al., "Selective vulnerability of preterm white matter to oxidative damage defined by F2-isoprostanes," Annals of Neurology, vol. 58, no. 1, pp. 108-120, 2005.

[24] R. L. Haynes, R. D. Folkerth, R. J. Keefe et al., "Nitrosative and oxidative injury to premyelinating oligodendrocytes in periventricular leukomalacia," Journal of Neuropathology \& Experimental Neurology, vol. 62, no. 5, pp. 441-450, 2003.

[25] Z. Huang, J. Liu, P.-Y. Cheung, and C. Chen, "Long-term cognitive impairment and myelination deficiency in a rat model of perinatal hypoxic-ischemic brain injury," Brain Research, vol. 1301, pp. 100-109, 2009.

[26] Y.-P. Deng, Y. Sun, L. Hu et al., "Chondroitin sulfate proteoglycans impede myelination by oligodendrocytes after perinatal white matter injury," Experimental Neurology, vol. 269, pp. 213-223, 2015.

[27] G. Huguet, E. Ey, and T. Bourgeron, "The genetic landscapes of autism spectrum disorders," Annual Review of Genomics and Human Genetics, vol. 14, no. 1, pp. 191-213, 2013.

[28] V. Cvetkovska, A. D. Hibbert, F. Emran, and B. E. Chen, "Overexpression of Down syndrome cell adhesion molecule impairs precise synaptic targeting," Nature Neuroscience, vol. 16, no. 6, pp. 677-682, 2013.

[29] A. Pitkänen, R. Roivainen, and K. Lukasiuk, "Development of epilepsy after ischaemic stroke," The Lancet Neurology, vol. 15, no. 2, pp. 185-197, 2016.

[30] S. T. Ferreira, M. V. Lourenco, M. M. Oliveira, and F. G. De Felice, "Soluble amyloid- $\hat{\mathrm{I}}^{2}$ oligomers as synaptotoxins leading to cognitive impairment in Alzheimerâ $€^{\mathrm{TM}} \mathrm{s}$ disease," Frontiers in Cellular Neuroscience, vol. 9, 2015.

[31] M. Sheng, B. L. Sabatini, and T. C. Sudhof, "Synapses and Alzheimer's disease," Cold Spring Harbor Perspectives in Biology, vol. 4, no. 5, Article ID a0057, 2012. 
[32] S. Janezic, S. Threlfell, P. D. Dodson et al., "Deficits in dopaminergic transmission precede neuron loss and dysfunction in a new Parkinson model," Proceedings of the National Academy of Sciences, vol. 110, no. 42, pp. E4016-E4025, 2013.

[33] T. Xu, X. Yu, A. J. Perlik et al., "Rapid formation and selective stabilization of synapses for enduring motor memories," Nature, vol. 462, no. 7275, pp. 915-919, 2009.

[34] J. A. Hirsch, X. Wang, F. T. Sommer, and L. M. Martinez, "How inhibitory circuits in the thalamus serve vision," $A n$ nual Review of Neuroscience, vol. 38, no. 1, pp. 309-329, 2015.

[35] M. M. Halassa and S. Kastner, "Thalamic functions in distributed cognitive control," Nature Neuroscience, vol. 20, no. 12, pp. 1669-1679, 2017.

[36] A. S. Mitchell, S. M. Sherman, M. A. Sommer, R. G. Mair, R. P. Vertes, and Y. Chudasama, "Advances in understanding mechanisms of thalamic relays in cognition and behavior," Journal of Neuroscience, vol. 34, no. 46, pp. 15340-15346, 2014.

[37] S. P. Miller and D. M. Ferriero, "From selective vulnerability to connectivity: insights from newborn brain imaging," Trends in Neurosciences, vol. 32, no. 9, pp. 496-505, 2009.

[38] E. McClendon, K. Chen, X. Gong et al., "Prenatal cerebral ischemia triggers dysmaturation of caudate projection neurons," Annals of Neurology, vol. 75, no. 4, pp. 508-524, 2014.

[39] I. Song and A. Dityatev, "Crosstalk between glia, extracellular matrix and neurons," Brain Research Bulletin, vol. 136, pp. 101-108, 2018.

[40] E. Dzyubenko, D. Manrique-Castano, C. Kleinschnitz, A. Faissner, and D. M. Hermann, "Role of immune responses for extracellular matrix remodeling in the ischemic brain," Therapeutic Advances in Neurological Disorders, vol. 11, Article ID 175628641881809, 2018.

[41] M. Stefaniuk, A. Beroun, T. Lebitko et al., "Matrix metalloproteinase- 9 and synaptic plasticity in the central amygdala in control of alcohol-seeking behavior," Biological Psychiatry, vol. 81, no. 11, pp. 907-917, 2017.

[42] X. Chen, Y. Liu, J. Zhu et al., "GSK-3 $\beta$ downregulates Nrf2 in cultured cortical neurons and in a rat model of cerebral ischemia-reperfusion," Scientific Reports, vol. 6, no. 1, 2016.

[43] C. Hooper, R. Killick, and S. Lovestone, "The GSK3 hypothesis of Alzheimer's disease," Journal of Neurochemistry, vol. 104, no. 6, pp. 1433-1439, 2008.

[44] P. K. Kamat, A. Kalani, S. Rai et al., "Mechanism of oxidative stress and synapse dysfunction in the pathogenesis of Alzheimer's disease: understanding the therapeutics strategies," Molecular Neurobiology, vol. 53, no. 1, pp. 648-661, 2016.

[45] F. Valtorta, M. Pennuto, D. Bonanomi, and F. Benfenati, "Synaptophysin: leading actor or walk-on role in synaptic vesicle exocytosis?," BioEssays, vol. 26, no. 4, pp. 445-453, 2004.

[46] F. Ackermann, C. L. Waites, and C. C. Garner, "Presynaptic active zones in invertebrates and vertebrates," EMBO Reports, vol. 16, no. 8, pp. 923-938, 2015.

[47] E. Kim and M. Sheng, "PDZ domain proteins of synapses," Nature Reviews Neuroscience, vol. 5, no. 10, pp. 771-781, 2004.

[48] Y. Shinohara, "Quantification of postsynaptic density proteins: glutamate receptor subunits and scaffolding proteins," Hippocampus, vol. 22, no. 5, pp. 942-953, 2012.

[49] R. L. Haynes, S. S. Billiards, N. S. Borenstein, J. J. Volpe, and H. C. Kinney, "Diffuse axonal injury in periventricular leukomalacia as determined by apoptotic marker fractin," Pediatric Research, vol. 63, no. 6, pp. 656-661, 2008.

[50] Y. D. Zhao, S. Ou, S. Y. Cheng et al., "Dendritic development of hippocampal CA1 pyramidal cells in a neonatal hypoxia- ischemia injury model," Journal of Neuroscience Research, vol. 91, no. 9, pp. 1165-1173, 2013.

[51] S. Won, S. Incontro, R. A. Nicoll, and K. W. Roche, "PSD-95 stabilizes NMDA receptors by inducing the degradation of STEP61," Proceedings of the National Academy of Sciences, vol. 113, no. 32, pp. E4736-E4744, 2016.

[52] I. E. Holopainen, "Seizures in the developing brain: cellular and molecular mechanisms of neuronal damage, neurogenesis and cellular reorganization," Neurochemistry International, vol. 52, no. 6, pp. 935-947, 2008. 


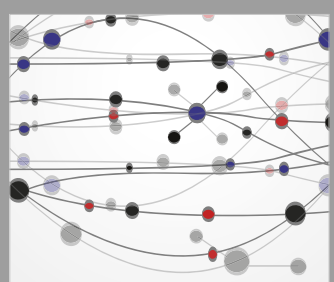

The Scientific World Journal
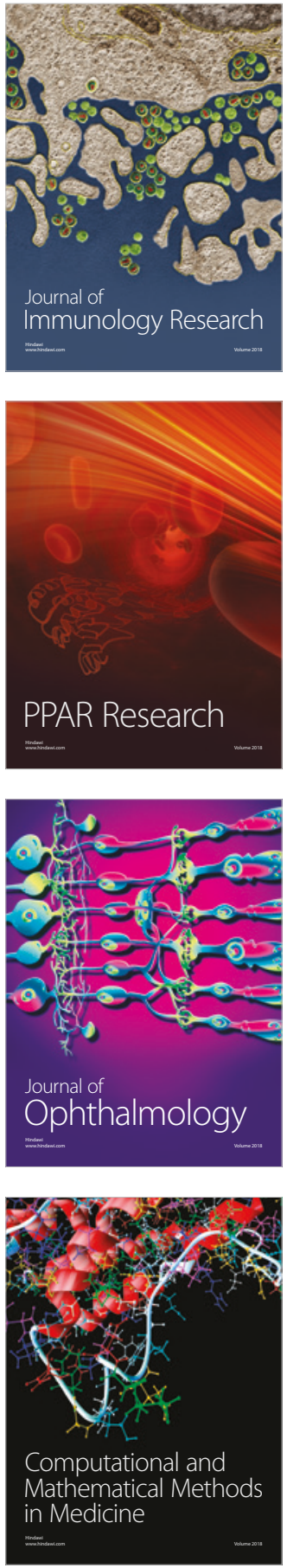

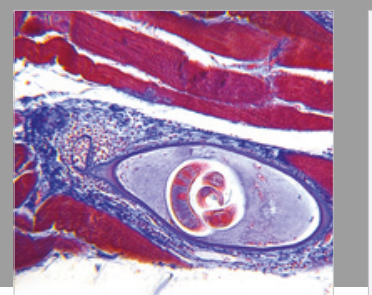

Gastroenterology Research and Practice

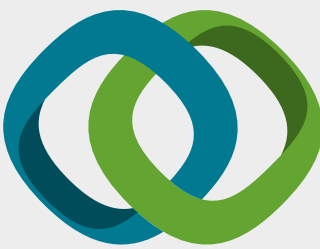

\section{Hindawi}

Submit your manuscripts at

www.hindawi.com
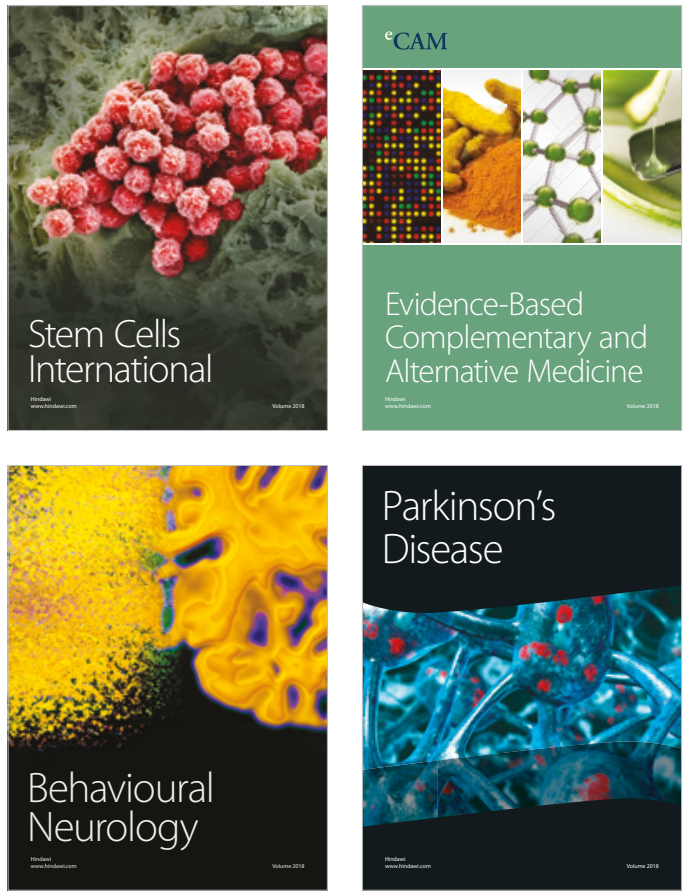

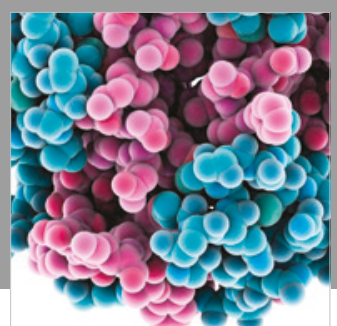

ournal of

Diabetes Research

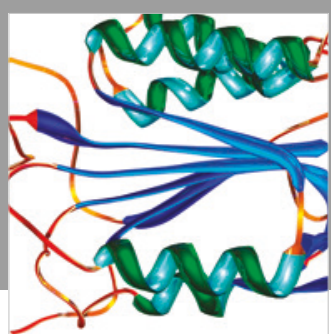

Disease Markers
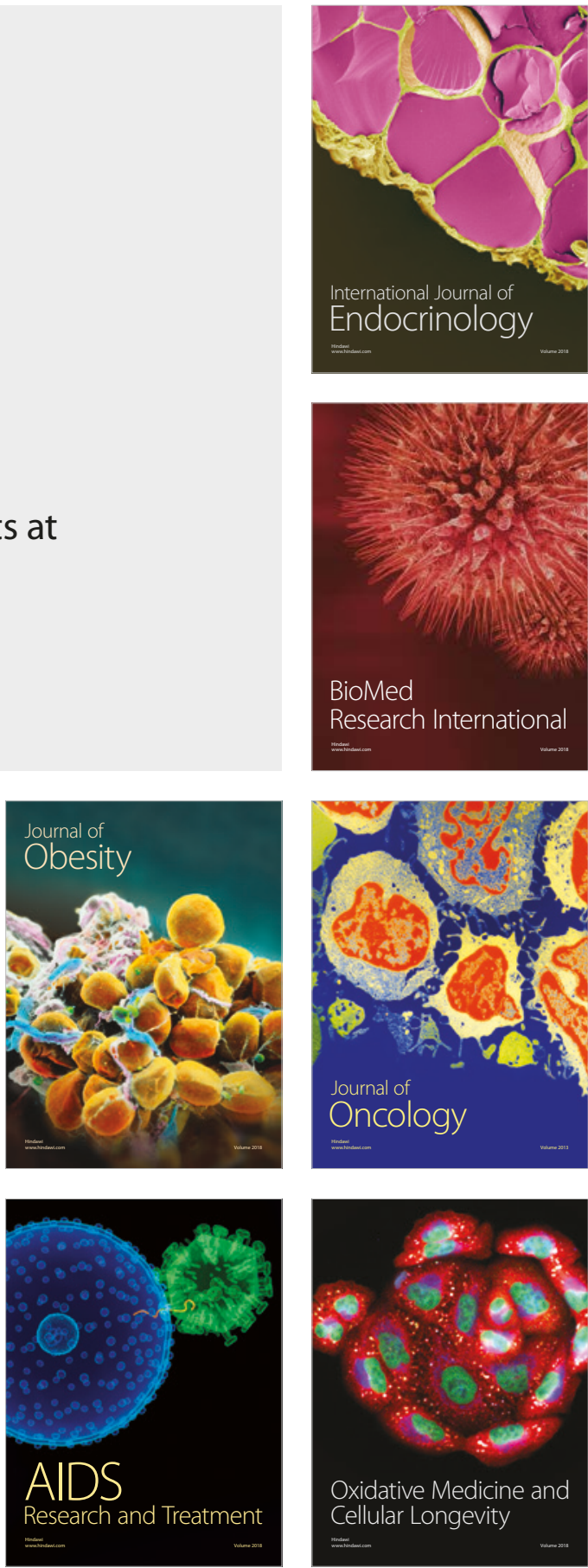